Inflammation and inflammatory bowel disease

\section{Uncoupling the p38 MAPK kinase in IBD: a double edged sword?}

\section{Arulampalam, S Pettersson}

\section{Dichotomal effects of specific p38 mitogen activated protein kinase (MAPK) inhibition in trinitrobenzene sulphonic acid (TNBS) induced colitis in mice}

U lcerative colitis (UC) and Crohn's disease $(C D)$, the most predominant manifestations of inflammatory bowel diseases (IBD), are accelerating major health problems. Although the aetiology of IBD remains elusive, a great deal of work has been carried out in successfully identifying some of the pathological and clinical profiles that are perturbed in these patients. This has led to the description of some of the molecular mechanisms that may underlie the idiopathic inflammation encountered in IBD. More recently, gene families and clusters which may be implicated in the onset of IBD, albeit in a limited manner, have been discovered.

Cytokines such as interleukin (IL)- 1 , IL-6, tumour necrosis factor $\alpha$ (TNF- $\alpha$ ), and interferon $\gamma$ among others, have been shown to maintain the highly chronic inflamed state of the bowel, if not actually causing the onset of this condition. Hence one (major) effort to uncouple the vicious circle and to combat this disease has been to eliminate or diminish the effects of these cytokines. The use of molecules blocking cytokine receptor interactions, by disrupting the means of cytokine activity (for example, inhibiting nuclear factor $\mathrm{\kappa B}$ using antisense molecules), is well documented. Taken together, there is an increasing demand to further understand the signalling mechanisms of various cytokines.

In most instances, the mitogen activated protein kinase (MAPK) family of enzymes are involved in transducing the cytokine signal within the epithelial cell of the gut mucosa. These enzymes are predominantly serine/threonine kinases and are activated via phosphorylation of these moieties in a well regulated cascade, leading to activation of specific transcription factors and subsequent target genes. Nevertheless, a linear cascade of kinase activation appears unlikely as other "peripheral" effectors most likely will also be induced and should therefore be included in any therapeutic regime directed towards cytokine signalling pathways.

A number of such anti-inflammatory therapies undergoing pharmacological and preclinical studies have targeted numerous serine/threonine kinases. Of these kinases, activation of p38 MAPK by a plethora of extracellular stimuli, including cellular stress, osmotic shock, and proinflammatory cytokines, is well documented. A number of p38 kinase inhibitors have been identified, including a dominant negative p38 molecule, but harnessing use of small molecule inhibitors appears to be the most promising in the present clinical setting.

Initially identified as an inhibitor of cytokine release (for example, TNF), the pyridinilimidazole compound SB203580 was later identified as an inhibitor of p38 kinase activity. This and other p38 inhibitors (for example, CNI-1493, ML3163) have been previously shown to be efficient in reducing the severity of inflammatory disease, including endotoxin induced mortality and collagen induced arthritis in mouse models. In this issue of Gut, ten Hove and colleagues ${ }^{1}$ have queried the efficacy of this compound in blocking trinitrobenzene sulphonic acid (TNBS) induced colitis [see page 507]. The results, although not entirely unexpected, have posed some interesting questions regarding p38 function, especially in IBD. Briefly, limited reduction in IL-12 cytokine production was observed, with no discernible loss of TNF expression in colon homogenates. Furthermore, in SB 203580 treated TNBS mice, wasting was more significant but colonic weight was higher despite a decrease in cell numbers in colonic lymph nodes compared with controls. Thus one of the observed effects may be to intervene on cell fate, be it to stop cell growth or induce apoptosis. Hence in this instance it appears that although some of the inflammatory agents are affected, thus in part abrogating the inflammatory process, it is not sufficient to completely ameliorate inflammation by p38 inactivation.

A number of explanations for the lack in efficacy have been suggested. One explanation assumes that "disease" initiating signalling pathways, bypassing the p38 block, precede p38 activation. Another invokes the function of upstream activators which can regulate inflammation in a p38 independent manner in TNBS induced colitis.

The precise nature of colitis induction by TNBS is unknown and thus other "inflammatory pathways" could also be activated by such an agent. To date, at least five isoforms of p38, two of which are ubiquitous, have been identified. Although regarded as a selective inhibitor of p38, extensive studies have not revealed a pan isoform inhibition through SB 203580. Hence some of the effects seen in this study may be due to the nature of the inhibitor, primarily those pertaining to antiproliferation and cell cycle regulation. The results presented by ten Hove et al are therefore of general interest and increased research activity in this area is certainly much needed.

One other alternative explanation is the less studied role of p38 induced downregulation of signalling. In the absence of an active p38 MAPK, negative feedback regulation of inflammatory signalling may well be hampered. In these instances, not only intercellular (as discussed by ten Hove and colleagues ${ }^{1}$ ) but also intracellular cross talk may be affected. At present the nature of the latter remains to be elucidated but given the importance of switch off mechanisms, this field of enquiry will no doubt receive warranted attention in the near future.

IBD being multifactorial speaks for a complexity that transcends the perturbation of just one (p38) among many kinases. Hence in the gut, merely inactivating p38 may not be sufficient to ameliorate the inflammatory process. Alternatively, p38 may still have a kinase independent role (as postulated for Jak2), involved in recruitment and structural modification. This function may contribute to the enhanced severity of the disease in this study as p38 itself is still phosphorylated.

The work presented by ten Howe et al in this issue of $G u t^{1}$ illuminates the complexity of signalling mechanisms that must be unravelled before we can obtain a coherent picture of diseases such as IBD. Having acquired this information, but only then, can the development of new drugs be expected. Until then the question as to whether deactivating p38 suffices in ameliorating (TNBS induced) colitis remains to be answered.

Gut 2002;50:446-447 


\section{A....................}

Authors' affiliations

V Arulampalam, S Pettersson, Microciology

and Tumorbiology Centre, Karolinska Institute,

Stockholm, Sweden
Correspondence to: Professor Sven Pettersson, Centre for Genomics Research, Karolinska Institute, Doktorsringen 2P, 17177 Stockholm, Sweden, sven.pettersson@cgr.ki.se

\section{REFERENCE}

1 ten Hove T, van den Blink B, Pronk I, et al. Dichotomal role of inhibition of p38 MAPK with SB 203580 in experimental colitis. Gut 2002;50:507-12.

\section{Homeobox genes: going for growth}

\section{R J Playford}

\section{The role of homeobox genes and dietary factors in gut growth and differentiation}

n this issue of Gut, Domon-Dell and colleagues $^{1}$ report the influence of butyrate on the intestinal homeobox gene $C d \times 2$ [see page 525]. Butyrate is a byproduct of fibre fermentation by colonic bacteria and is known to be a substrate for colonocytes. Over the last few years there has been increasing interest in the function of genes involved in controlling the anatomical relationships between various organs. This article therefore touches on the role of homeobox genes and dietary factors in gut growth and differentiation.

The development of multicellular animals is dependent on expression of a hierarchy of genes that sequentially provide increasingly detailed positional information. Many of these genes contain a highly preserved "homeobox" sequence that codes for a DNA binding homeo domain. In insects, a group of such genes-specifying the individual characteristics of body segments and known as homeotic selector genes of the Antp-type (the defining gene is named Antennapaedia) - are clustered in a complex known as the HOM cluster. These are expressed topographically in the same order as they occupy in chromosomal DNA. They are conserved strongly during evolution so that their homologues appear as four paraologous gene complexes known as Hox complexes which specify positional information in mammalian embryos.

Other homeobox genes are scattered in the genome and of particular interest are homologues of a Drosophila homeobox gene called Caudal (Cad). This is known to be important in determining anatomical positioning as Drosophila larvae lacking Cad gene products show the phenotype of deletions of many of the posterior segments. The first mammalian Cad homologue $(C d x I)$ was isolated from an embryonic mouse cDNA library with two further members of the Caudal family $(C d \times 2$ and $C d x 4)$ having subsequently been cloned in mice. $C d x 3$ was originally identified in the pancreas of hamsters but has subsequently been shown to be identical to $C d x 2$.

Mutation of $C d x I$ or 2 results in a skeletal homeotic shift, supporting an important role of the Cad genes in axial patterning. Homozygous deletion of $C d x 2$ is a lethal mutation, probably relating to its importance in the development of extra-embryonic tissue. The initial reis, heterozygote) mice considered that it resulted in distal bowel tumorigenesis, ${ }^{2}$ suggesting a role for $C d x 2$ as a tumour suppressor gene. More recently, additional studies suggest that this may have been a histological misinterpretation with the "tumours" being areas of gastric metaplasia. ${ }^{3}$ It therefore seems likely that $C d \times 2$ directs endodermal tissue towards a caudal differentiation and that $C d \times 2$ haplo insufficient areas develop as forestomach epithelium. Intercalary growth subsequently fills in the missing tissue types at the discontinuity between gastric and colonic epithelium resulting in the polypoidal lesions seen.

In addition to the key role that homeobox genes play during development, it is likely that at least some of these genes also play important functions on proliferation and differentiation during adult life. Results of manipulating Cdx-1 expression have given somewhat contradictory results. Transfection studies overexpressing Cdx-1 in the intestinal cell line, IEC6, has been reported to increase proliferation and differentiation ${ }^{4}$ but also transformational and tumorigenic activity. ${ }^{5}$ However, when the same approach was used on the human colonic cell lines $\mathrm{HT}_{2} 9^{6}$ or $\mathrm{Caco} 2$ cells, $^{7}$ it had virtually no effect on proliferation when transfected on its own but enhanced the growth inhibitory effect of $\mathrm{Cdx}-2$ in ports of the phenotype of $C d x 2+/-$ (that cotransfected cells. ${ }^{6}$ An alternative approach is to reduce the endogenous levels of Cdx-l using antisense RNA This has been tried and shown to slow growth of Caco-2 cells, ${ }^{7}$ providing additional support for the idea that $\mathrm{Cdx}-1$ plays a role in controlling proliferation. The data in support of Cdx-2 functioning as a tumour suppressor gene are somewhat stronger: $\mathrm{Cdx} 2$ overexpression reduces cell growth in IEC, Caco-2, and HT29 cells and Cdx2 expression is decreased in relation to the tumour grade in human colorectal cancers cells and in chemically induced tumours in the rat. ${ }^{8}$ In addition, the morphology of transfected IEC and Caco-2 cells is altered with increased differentiation and a rise in expression of small intestinal digestive enzymes such as sucraseisomaltase. $^{7}$ Taken together, it appears likely that $\mathrm{Cdx} 2$ functions as a regulator of intestinal cell differentiation in addition to its developmental role in embryogenesis.

Control of Cdx-1 and 2 expression is poorly understood although alteration in oncogenic Ras activation, which is an early and frequent event in colorectal cancers, is thought to decrease Cdx-2 expression, acting via the $P K C$-jun-fos pathway while increasing Cdx-1 expression by acting through the Raf-MEKl pathway (for an excellent review, see Freud and colleagues ${ }^{9}$ ). In the paper reported in this issue of Gut, Domon-Dell et al have focused on the interactions between Cdx-2 expression and butyrate and found that its presence stimulates Cdx-2 expression. In their discussion, they provide further (unpublished) information regarding potential mechanisms for this effect, such as the possibility of an atypical butyrate response element by which it might act at a molecular level although the final answer remains unclear. Whichever mechanisms are involved, it seems likely that this is an example of direct nutrient regulation of intestinal cell function and its findings have applicability beyond the current study.

The usual impression of a "growth factor" is a peptide that binds to specific external receptors, inducing a secondary signalling cascade resulting in cell proliferation. When considering complex interactions, such as the control of growth and differentiation of gut cells, it is important to appreciate that the somewhat arbitrary labelling of a molecule to 
an individual function-for example, considering "epidermal growth factor" simply as a stimulant of proliferationcan be misleading as it is now clear that such factors have multiple effects. For example, although they are often considered separately, the distinction between cytokines and growth factors are sometimes blurred as the "cytokine" interleukin 8 has been shown to stimulate migration of human colonic epithelial cells, ${ }^{10}$ a process normally associated with "growth factors", and peptides normally considered to be "growth factors" can influence immunological function. Similarly, molecules such as glutamine or butyrate which have been generally considered to be simple energy providers, and vitamins such as A and D, which were at one time thought to have limited biological functions, are now known to influence many other activities of the cell, such as development, differentiation, and proliferation (for example, see Nagpal and Chandraratna ${ }^{11}$ ). The current study therefore provides further evidence for additional actions of relatively simple molecules on multiple functions within a cell, acting through pathways such as specific DNA response elements. The distinction between nutrition and pharmacology is therefore less clear than it first appears.

Gut 2002;50:447-448

\section{Author's affiliation}

R J Playford, Gastroenterology Section, Department of Medicine, Imperial College Faculty of Medicine, Hammersmith Hospital Campus, London, UK

Correspondence to: Professor R J Playford, Department of Gastroenterology, Hammersmith Hospital, Du Cane Rd, London W12 ONN, UK r.playford@ic.ac.uk

\section{REFERENCES}

1 Domon-Dell C, Wang Q, Kim S, et al. Stimulation of the intestinal $\mathrm{Cdx} 2$ homeobox gene by butyrate in colon cancer cells. Gut 2002:50:525-9.

2 Chwengsaksophak KJR, Hammond VE, Kontgen $\mathrm{F}$, et al. Homeosis and intestinal tumours in $\mathrm{Cdx} 2$ mutant mice. Nature 1997;385:84-7.

3 Beck F, Chwengsaksophak K, Waring P, et al. Reprogramming of intestinal cell differentiation and intercalary regeneration in Cdx2 mutant mice. Proc Natl Acad Sci USA 1999;96:7318-23.

4 Soubeyran P, Andre F, Lissitzy JC, et al. $\mathrm{Cdx} l$ promotes differentiation in a rat intestinal epithelial cell line. Gastroenterology 1999;117:1326-38.

5 Soubeyran P, Haglund K, Garcia S, et al. Homeobox gene $\mathrm{Cdx} 1$ regulates Ras, Rho and PI3 kinase pathways leading to transformation and tumorigenesis of intestinal epithelial cells. Oncogene 2001;20:4180-7.

6 Mallo GV, Soubeyran P, Lissitzky JC, et al. Expression of $\mathrm{Cdx} 1$ and $\mathrm{Cd} 22$ homeotic genes leads to reduced malignancy in colon cancer-derived cells. J Biol Chem 1998;273:14030-6.

7 Lorentz O, Duluc I, Arcangelis AD, et al. Key role of $\mathrm{Cdx} 2$ homeobox gene in extracellular matrix-mediated intestinal cell differentiation. J Cell Biol 1997; 139:1553-65.

8 Ee HC, Erler T, Bhathal PS, et al. Cdx2 homeodomain protein expression in human and rat colorectal adenoma and carcinoma. Am J Pathol 1995;147:586-92.

9 Freud JN, Domon-Dell C, Kedinger $M$, et al. The $\mathrm{Cdx} 1$ and $C d \times 2$ homeobox genes in the intestine. Biochem Cell Biol 1998;76:957-69.

10 Wilson AJ, Gibson PR. Epithelial migration in the colon: filling in the gaps. Clin Sci 1997;93:97-108.

11 Nagpal S, Chandraratna RA. Vitamiin A and regulation of gene expression. Curr Opin Clin Nutr Metab Care 1998; 1:341-6.

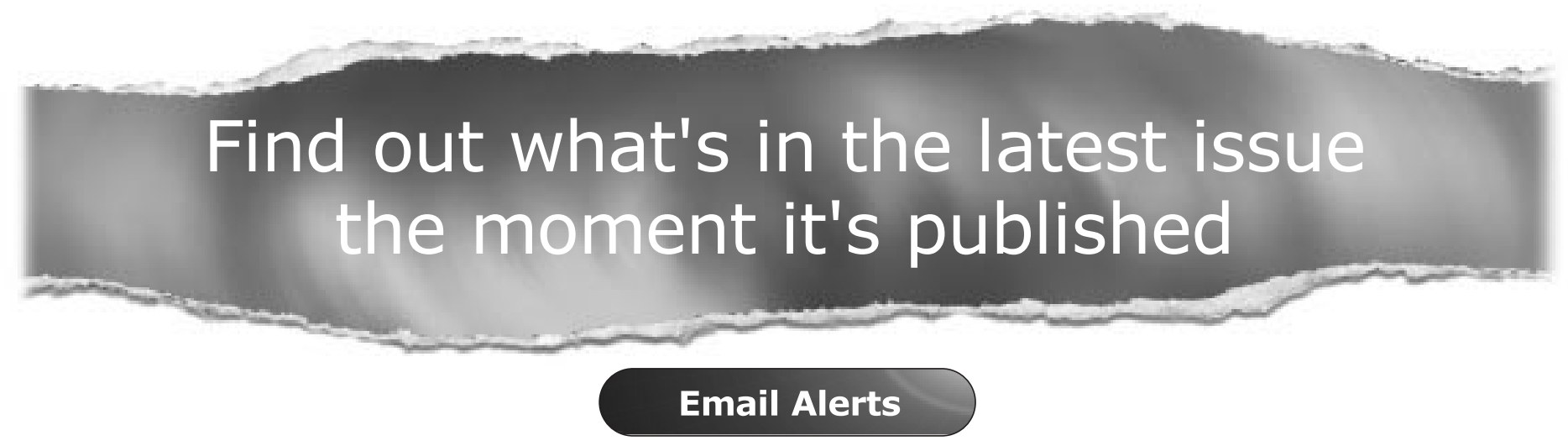

Sign up to receive the table of contents by email every month. You can select from three alerts: Table of Contents (full), TOC Awareness (notice only); Gut related announcements.

www.gutjnl.com 